\title{
The Application of Crisis Intervention in Middle-Aged and Young Patients with Acute Myocardial Infarction after PCI
}

\author{
Caiyun Weng, Chunmei Gao, Jianjing Chen \\ Department of Cardiovascular Medicine, Affiliated Haian Hospital of Nantong University, Jiangsu, China
}

\section{ABSTRACT}

Objective: To investigate the effect of crisis intervention in middle-aged and young patients with acute myocardial infarction after percutaneous coronary stent implantation (PCI).

Methods: A total of 108 middle-aged and young patients with acute myocardial infarction undergoing PCI were selected from July 2018 to July 2019 in the Department of Cardiology, Hai'an County People's Hospital. They were divided into two groups, according to a random number table, with 54 cases in each group. The control group implemented routine postoperative intervention, and the intervention group implemented postoperative crisis intervention. The changes in the two groups' sense of crisis, mental state, quality of life and hope level before and after the intervention were compared.

Results: The emotional, behavioral, and cognitive scores of the intervention group were lower than those of the control group after 4 weeks of intervention $(P<.05)$. The mental state scores of the intervention group were lower than those of the control group $(P<.05)$. Also, the various quality of life scores were higher than those of the control group $(P<.05)$. The intervention group's hope level scores were higher than the control group $(P<.05)$ after 4 weeks of intervention.

Conclusion: The application of crisis intervention to middle-aged and young patients with acute myocardial infarction after PCI can reduce the sense of crisis, improve their mental state and quality of life, and raise the level of hope.

\section{INTRODUCTION}

Acute myocardial infarction, as a type of coronary heart disease, has high morbidity and mortality [Zhang 2020]. It is caused by coronary artery stenosis caused by coronary atherosclerosis, which leads to myocardial ischemia and necrosis, and finally leads to disease. Coronary heart disease can induce serious complications, such as malignant arrhythmia, heart failure, and sudden death [Plakht 2020; Boinska 2020]. PCI is an important clinical treatment for acute myocardial infarction at present; it comes with less trauma and a high success rate of operation. However, because of the high operation

Received March 5, 2021; received in revised form March 30, 2021; accepted March 30, 2021.

Correspondence: Caiyun Weng, Department of Cardiovascular Medicine, Hai'an Hospital Affiliated to Nantong University, Nantong, Fiangsu, 226600, China. cost, puncture pain and long-term bed rest after operation, it is easy for patients to suffer from anxiety, fear and other bad emotions, which eventually will lead to high-risk accidents after PCI [Shiyovich 2019; Worcester 2019]. Compared with elderly patients with acute myocardial infarction, young and middle-aged patients are more likely to have greater stress due to illness because of taking on more family responsibilities and social roles [Pandey 2020]. Crisis intervention is a common psychosocial intervention in foreign countries in recent years, which can fully mobilize the potential of patients in crisis and overcome psychological crisis by rebuilding their healthy psychology before crisis [Twigg 2019]. In this study, crisis intervention was given to young and middle-aged patients with acute myocardial infarction after PCI in our hospital, and the effect of routine intervention was compared. The results are shown below.

\section{DATA AND METHODS}

\section{General data from fuly 2018 to Fuly 2019}

One-hundred-eight young and middle-aged patients with acute myocardial infarction undergoing PCI were selected and divided into two groups, according to random number table method, with 54 cases in each group. There were 31 males and 23 females in the control group. The average age was (42.95 6.17 ) years old. Infarction sites: 23 cases of inferior wall, 15 cases of anterior wall, nine cases of anterior septal wall infarction, and seven cases of positive posterior wall infarction. Education level: 11 cases in primary schools, 28 cases in middle schools, and 15 cases in universities. There were 30 males and 24 females in the intervention group. The average age was (41.88 6.25) years old. Infarction sites: 24 cases of inferior wall, 16 cases of anterior wall, eight cases of anterior septal wall infarction, and six cases of positive posterior wall infarction. Education level: 13 cases in primary schools, 25 cases in middle schools, and 16 cases in universities. Inclusion criteria: Meet the diagnostic criteria of acute myocardial infarction. PCI successfully was performed within 24 hours of admission. The age is $18-59$ years old. Clear consciousness; have normal communication skills; and condition is stable. Exclusion criteria: There is mental abnormality or cognitive dysfunction. After operation, the cardiac function was classified as grade IV by KilliP. Also excluded were cases of cardiogenic shock or those with incomplete clinical data. This study was approved by the hospital ethics committee. There was no significant difference in general data between the two groups $(P>.05)$. 
Table 1. Comparison of the sense of crisis between the two groups before and after intervention ( $\bar{x} \pm s, s c o r e)$

\begin{tabular}{lccc}
\hline Time & Group & Emotion & Behavior \\
\hline Before intervention & Control group $(\mathrm{N}=54)$ & $7.57 \pm 1.02$ & $7.51 \pm 0.88$ \\
$\quad$ Intervention group $(\mathrm{N}=54)$ & $7.61 \pm 0.99$ & $7.55 \pm 0.91$ & $7.10 \pm 0.89$ \\
T value & 0.207 & 0.232 & 0.173 \\
P-value & .837 & .817 & .863 \\
After 4 weeks of intervention & Control group $(\mathrm{N}=54)$ & $5.45 \pm 0.71$ & $5.92 \pm 0.61$ \\
Intervention group $(\mathrm{N}=54)$ & $4.03 \pm 0.69$ & $5.08 \pm 0.57$ & $5.22 \pm 0.51$ \\
T value & 10.540 & 7.394 & 6.192 \\
P-value & .000 & .000 & .000
\end{tabular}

Table 2. Comparison of mental state between the two groups before and after intervention ( $\overline{\mathrm{x}} \pm \mathrm{s}$, score)

\begin{tabular}{|c|c|c|c|c|c|c|c|c|c|c|}
\hline Before intervention & $\begin{array}{l}\text { Control group } \\
\qquad(\mathrm{N}=54)\end{array}$ & $\begin{array}{c}2.97 \pm \\
0.50\end{array}$ & $\begin{array}{l}3.05 \pm \\
0.59\end{array}$ & $3.17 \pm 0.38$ & $2.79 \pm 0.45$ & $\begin{array}{c}2.88 \pm \\
0.41\end{array}$ & $\begin{array}{c}2.77 \pm \\
0.51\end{array}$ & $\begin{array}{c}2.94 \pm \\
0.56\end{array}$ & $\begin{array}{c}2.57 \pm \\
0.44\end{array}$ & $\begin{array}{c}2.79 \pm \\
0.56\end{array}$ \\
\hline $\begin{array}{l}\text { Intervention group } \\
(\mathrm{N}=54)\end{array}$ & $3.01 \pm 0.52$ & $\begin{array}{c}3.02 \pm \\
0.56\end{array}$ & $\begin{array}{l}3.19 \pm \\
0.43\end{array}$ & $2.75 \pm 0.48$ & $2.79 \pm 0.45$ & $\begin{array}{c}2.80 \pm \\
0.55\end{array}$ & $\begin{array}{c}2.98 \pm \\
0.51\end{array}$ & $\begin{array}{c}2.61 \pm \\
0.47\end{array}$ & $\begin{array}{c}2.77 \pm \\
0.52\end{array}$ & \\
\hline$T$ value & 0.407 & 0.271 & 0.256 & 0.447 & 1.086 & 0.294 & 0.388 & 0.457 & 0.192 & \\
\hline$P$-value & .685 & .787 & .798 & .656 & .280 & .769 & .699 & .649 & .848 & \\
\hline After intervention & $\begin{array}{l}\text { Control group } \\
\qquad(\mathrm{N}=54)\end{array}$ & $\begin{array}{c}2.81 \pm \\
0.52\end{array}$ & $\begin{array}{l}2.91 \pm \\
0.63\end{array}$ & $2.85 \pm 0.57$ & $2.37 \pm 0.59$ & $\begin{array}{c}2.18 \pm \\
0.49\end{array}$ & $\begin{array}{c}2.21 \pm \\
0.47\end{array}$ & $\begin{array}{c}2.19 \pm \\
0.50\end{array}$ & $\begin{array}{l}1.99 \pm \\
0.39\end{array}$ & $\begin{array}{l}1.89 \pm \\
0.55\end{array}$ \\
\hline $\begin{array}{l}\text { Intervention group } \\
(\mathrm{N}=54)\end{array}$ & $1.39 \pm 0.49$ & $\begin{array}{l}1.59 \pm \\
0.54\end{array}$ & $\begin{array}{c}1.26 \pm \\
0.50\end{array}$ & $1.40 \pm 0.39$ & $1.33 \pm 0.35$ & $\begin{array}{l}1.47 \pm \\
0.51\end{array}$ & $\begin{array}{l}1.36 \pm \\
0.45\end{array}$ & $\begin{array}{l}1.52 \pm \\
0.43\end{array}$ & $\begin{array}{l}1.57 \pm \\
0.59\end{array}$ & \\
\hline
\end{tabular}

\section{Methods}

The control group was given routine intervention, and the interveners told the patients in detail about the knowledge of acute myocardial infarction and PCI and the daily healthy diet guidance. These patients received follow up by telephone after the operation. The intervention group implemented crisis intervention as follows: (1) The first step was to establish a crisis intervention team. Team members are specialists, head nurses, psychological counselors, and specialist nurses. All members have rich clinical experience and good communication skills. Organize team members to learn about PCI operation, and carry out training on psychological crisis intervention and evaluation scale. (2) Evaluate psychological problems. Interveners explained the detailed knowledge of acute myocardial infarction and PCI to patients in detail, combined with PPT, video education, and pictures. Interveners fully understand and evaluate the psychological crisis of patients after PCI and communicate with patients one-on-one after PCI to master the psychological problems of patients. (3) Make a crisis intervention plan. Interveners make targeted intervention plans, according to each patient's condition, age, psychological status, and education level. Make sure not to pay attention to the shaping of the patient's personality, but to help the patient's psychological balance level before the crisis in the recovery period. At this stage, the interveners should learn more about the degree of damage caused by psychological crisis to patients' daily life and its impact on others and the surrounding environment, and at the same time fully affirm the strengths of patients, define the family and social support systems that can help patients, and define the treatment objectives. (4) Psychological support intervention. Psychological support is four-pronged. First, with empathy communication, the psychological state and existing psychological barriers of patients are deeply analyzed. Interveners actively communicate with patients, guide them to express their inner feelings, and give them psychological support by means of suggestion and empathy. Second, it is important to provide opportunities for drainage. Interveners encourage patients to fully express their feelings, recognize their true feelings and establish empathy, while not persuading them to change their feelings. The third step is explanation and guidance. Intervention personnel give patients daily diet and activity 
Table 3. Comparison of quality of life between the two groups before and after intervention $\bar{x}$ s, minute)

\begin{tabular}{lcccc}
\hline Time & Group & Somatic function & Social function & Psychological function \\
\hline Before intervention & Control group $(\mathrm{N}=54)$ & $12.19 \pm 3.01$ & $11.78 \pm 2.25$ & $15.37 \pm 3.35$ \\
$\quad$ Intervention group $(\mathrm{N}=54)$ & $12.26 \pm 2.97$ & $11.83 \pm 2.07$ & $15.51 \pm 3.61$ & $12.55 \pm 2.37$ \\
T value & 0.122 & 0.120 & 0.209 & 0.160 \\
P-value & .903 & .905 & .835 & .874 \\
After intervention & Control group $(\mathrm{N}=54)$ & $15.13 \pm 3.76$ & $13.04 \pm 3.55$ & $20.16 \pm 4.48$ \\
Intervention group $(\mathrm{N}=54)$ & $21.03 \pm 4.71$ & $18.95 \pm 3.82$ & $28.57 \pm 5.15$ & $24.27 \pm 5.27$ \\
T value & 7.194 & 8.328 & 9.054 & 6.705 \\
P-value & .000 & .000 & .000 & .000
\end{tabular}

Table 4. Comparison of hope level between the two groups before and after intervention $\bar{x}$ s, score)

\begin{tabular}{lcccc}
\hline Time & Group & Take positive action & Keep close to others & A positive attitude toward reality and the future \\
\hline Before intervention & Control group $(\mathrm{N}=54)$ & $9.11 \pm 1.03$ & $11.09 \pm 1.13$ & $9.03 \pm 1.01$ \\
Intervention group $(\mathrm{N}=54)$ & $9.06 \pm 0.97$ & $11.21 \pm 1.19$ & $9.09 \pm 1.04$ & 0.304 \\
T value & 0.260 & 0.537 & 0.762 & $10.13 \pm 1.18$ \\
P-value & 0.796 & 0.592 & $12.25 \pm 1.24$ & $13.02 \pm 1.21$ \\
After intervention & Control group $(\mathrm{N}=54)$ & $10.26 \pm 1.13$ & 12.565 & 0.000
\end{tabular}

guidance after operation, help them establish correct cognition and explain the process of crisis occurrence and development, so that they can face the disease correctly and actively participate in the postoperative rehabilitation process. Interveners set up WeChat group, and encouraged patients to communicate with each other in WeChat group with "Home of Patients with Acute Myocardial Infarction." Interveners answered patients' questions in time. The fourth step is to improve self-confidence. Interveners provide patients with an effective psychological crisis coping model to enhance their self-confidence, fully affirm their progress in the process of cardiac rehabilitation, help patients relieve negative emotions by humor and altruism, and instruct patients to record their self-rehabilitation and psychological state changes every day. Finally, the last step is social support system intervention. Interveners introduce the importance of family and social support and its effect on stress relief to patients and their families in detail, encourage patients' families and friends to communicate with patients, and help solve physical and mental stress caused by diseases, surgery and other factors. Interveners guide patients to make clear their role in the process of rehabilitation and make rational use of family and social support.

\section{Observation indicators}

(1) Sense of crisis: It is evaluated by TAF, which has three dimensions, with 1-10 points for each dimension. The lower the score, the lower the sense of crisis [Sen 2019]. (2) Mental state: It is evaluated by SCL-90, which has nine dimensions, including forced symptoms, depression, somaties, interpersonal relationships, anxiety, hostilities, paranies, horror, mental, total 90 entries, with 0-4 points in each dimension. The higher the score, the worse the mental state [Liu 2020]. (3) Quality of life: It is evaluated by the Comprehensive Quality of Life Rating Scale (GQOL-74), which consists of four dimensions, including physical functions, social functions, psychological functions, material life, a total of 20 items, each item has a score of $1-5$, with a total score of 20-100. The higher the score, the better the quality of life [Plakht 2020]. (4) Hope level: It is evaluated by Herth Hope Scale, which consists of three items and 12 items, with 1-4 points for each item and a total score of 12-48 points. The higher the score, the higher the hope level [Poursafar 2019].

SPSS22.0 was used for statistical analysis. Before and after intervention, the sense of crisis, mental state, quality of life and hope level of the two groups were expressed by $\overline{\mathrm{X}} \pm \mathrm{S}$, T test was expressed by $\mathrm{n}(\%)$, and $\chi^{2}$ test was used. $P<.05$ was statistically significant.

\section{RESULTS}

See Table 1 for the sense of crisis. The scores of emotion, behavior and cognition in the intervention group were lower than those in the control group $(P<.05)$ (Table 1$)$. 
Table 2 details psychological state. After 4 weeks of intervention, the scores of psychological state in the intervention group were lower than those in the control group $(P<.05)$ (Table 2$)$.

See Table 3 for the quality of life. The scores of quality of life in the intervention group were higher than those in the control group after 4 weeks of intervention $(P<.05)$ (Table 3$)$.

Table 4 outlines the desired level. After 4 weeks of intervention, the scores of hope levels in the intervention group were higher than those in the control group $(P<.05)$ (Table 4$)$.

\section{DISCUSSION}

Coronary heart disease (CHD), as one of the major cardiovascular diseases to endanger human health, has become the leading cause of death in many countries around the world [Sharif 2020]. Acute myocardial infarction (AMI) is one of the most harmful and fatal diseases in coronary heart disease [Köppen 2018]. In recent years, the incidence of acute myocardial infarction significantly has increased and has become one of the major diseases that threaten human health [Köppen 2018].

PCI is an effective treatment for acute myocardial infarction, but most patients will have adverse psychological emotions, due to long-term bed rest, puncture pain, and the risk of stent stenosis after operation [D'Rosario 2020]. As the pillar of society and family, middle-aged and young people are more likely to have anxiety, depression and other emotions, which affect the treatment and postoperative recovery. In this study, the implementation of crisis intervention in young and middle-aged patients with acute myocardial infarction after PCI in our hospital has achieved significant results.

The results of this study showed that after 4 weeks of intervention, the scores of emotion, behavior and cognition in the intervention group were lower than those in the control group, and the scores of psychological state in the intervention group were lower than those in the control group $(P<$ $0.05)$. It shows that crisis intervention for young and middleaged patients with acute myocardial infarction after PCI can effectively relieve psychological crisis and bad psychological mood. The main reasons for the analysis are that patients' lack of awareness of disease treatment and prognosis-related knowledge is the main reason for their psychological crisis. If patients are always in a negative emotional state and cannot be dispatched effectively, the psychological crisis will persist and gradually develop further, while patients' lack of family and social support will make it difficult for patients to eliminate the psychological crisis with external force [Moghaddam 2018; Plakht 2018]. Through crisis intervention, we can take solving patients' psychological crisis as the focus and goal of intervention and implement systematic and comprehensive intervention, according to the root causes and related influencing factors of patients' psychological crisis. This will help correct patients' misconceptions, guide them to vent their bad emotions reasonably, and assist patients to establish correct healthy behaviors, making full use of family and social support and quickly accepting the patients' role and the influence of formal illness and surgery on themselves. Thus, this effectively alleviates people's sense of psychological crisis and bad psychological emotions [Silber 2017; Bårdsgjerde 2020]. In this study, the scores of quality of life and hope level in the intervention group were higher than those in the control group after 4 weeks intervention $(P<.05)$. It shows that crisis intervention can effectively improve the quality of life and hope level of patients with acute myocardial infarction. The main reason for the analysis is that the interveners actively build a bridge of full trust with the patients during the crisis intervention, understand the psychological problems of the patients, give targeted interventions, help analyze the problems existing in their rehabilitation process, and set reasonable goals, which eventually will prompt patients to change their traditional wrong ideas, improve their psychological adaptability and tolerance, and actively participate in early postoperative rehabilitation, thus effectively improving their quality of life [Suzuki 2018]. In addition, by instructing patients to record their self-rehabilitation and psychological state changes every day and establishing a WeChat group, they can promote communication among patients, so that they can find problems themselves and solve problems with the help of medical staff and patients, so that they are full of confidence in physical rehabilitation after treatment, and finally effectively improve their hope level [Zullo 2020].

To sum up, the application of crisis intervention in young and middle-aged patients with acute myocardial infarction after PCI can reduce the sense of crisis, improve their psychological state and quality of life, and raise their hope level.

\section{ACKNOWLEDGEMENTS}

This work was supported by the Nantong Scientific Research Program Project (Grant No. MSZ18060) and by the Nantong Scientific Research Program Project (Grant No. MSZ18172).

\section{REFERENCES}

Bårdsgjerde EK, Landstad BJ, Hole T, et al. 2020. Nurses' perceptions of patient participation in the myocardial infarction pathway [J]. Nurs Open. 7(5):1606-1615.

Boinska J, Koziński M, Kasprzak M, et al. 2020. Diurnal variations in tissue factor and tissue factor pathway inhibitor concentrations in relation to on-treatment platelet reactivity: an analysis of patients with acute myocardial infarction [J]. Platelets. 31(7):877-883.

D'Rosario D, Currey J, Considine J, et al. 2020. Clinical deterioration in patients with ST-elevation myocardial infarction during and for 24 $\mathrm{h}$ after percutaneous coronary intervention: An observational study [J]. Aust Crit Care. 33(5):458-462.

Köppen J, Maier CB, Busse R, et al. 2018. What are the motivating and hindering factors for health professionals to undertake new roles in hospitals? A study among physicians, nurses and managers looking at breast cancer and acute myocardial infarction care in nine countries [J]. Health Policy. 122(10):1118-1125.

Liu H, Zhang J, Yu J, et al. 2020. Prognostic value of serum albuminto-creatinine ratio in patients with acute myocardial infarction: Results 
from the retrospective evaluation of acute chest pain study [J]. Medicine (Baltimore). 99(35):e22049.

Moghaddam N, Wong GC, Cairns JA, et al. 2018. Association of Anemia With Outcomes Among ST-Segment-Elevation Myocardial Infarction Patients Receiving Primary Percutaneous Coronary Intervention [J]. Circ Cardiovasc Interv. 11(12):e007175.

Pandey A, Keshvani N, Vaughan-Sarrazin MS, et al. 2020. Evaluation of Risk-Adjusted Home Time After Acute Myocardial Infarction as a Novel Hospital-Level Performance Metric for Medicare Beneficiaries [J]. Circulation. 142(1):29-39.

Plakht Y, Gilutz H, Arbelle JE, et al. 2020. Sex and Ethnic Disparities in Health-Related Outcomes Following Acute Myocardial Infarction in Israel [J]. Isr Med Assoc J. 22(5):303-309.

Plakht Y, Gilutz H, Shiyovich A. 2018. Sodium levels during hospitalization with acute myocardial infarction are markers of in-hospital mortality: Soroka acute myocardial infarction II (SAMI-II) project [J]. Clin Res Cardiol. 107(10):956-964.

Plakht Y, Greenberg D, Gilutz H, et al. 2020. Mortality and healthcare resource utilization following acute myocardial infarction according to adherence to recommended medical therapy guidelines [J]. Health Policy. 124(11):1200-1208.

Poursafar Z, Joukar F, Hasavari F, et al. 2019. The Associations between Meat Group Consumption and Acute Myocardial Infarction Risks in an Iranian Population: a Case-Control Study [J]. Clin Nutr Res. 8(2):159-168.

Sen A, Vardaxis I, Lindqvist BH, et al. 2019. Systematic assessment of prescribed medications and short-term risk of myocardial infarction - a pharmacopeia-wide association study from Norway and Sweden [J]. Sci Rep. 9(1):8257.
Sharif Nia H, Gorgulu O, Pahlevan Sharif S, et al. 2020. Prevalence of Acute Myocardial Infarction and Changing Meteorological Conditions in Iran: Fuzzy Clustering Approach [J]. Iran J Public Health. 49(5):923-930.

Shiyovich A, Axelrod M, Gilutz H, et al. 2019. Early Versus Late NewOnset Atrial Fibrillation in Acute Myocardial Infarction: Differences in Clinical Characteristics and Predictors [J]. Angiology. 70(10):921-928.

Silber H, Shiyovich A, Gilutz H, et al. 2017. hospital survivors of acute myocardial infarction. Soroka Acute Myocardial Infarction II (SAMI-II) project [J]. Int J Cardiol. 228:694-699.

Suzuki Y, Ito K, Yamamoto K, et al. 2018. Predictors of improvements in exercise capacity during cardiac rehabilitation in the recovery phase after coronary artery bypass graft surgery versus acute myocardial infarction [J]. Heart Vessels. 33(4):358-366.

Twigg DE, Kutzer Y, Jacob E, et al. 2019. A quantitative systematic review of the association between nurse skill mix and nursing-sensitive patient outcomes in the acute care setting [J]. J Adv Nurs. 75(12):3404-3423.

Worcester MU, Goble AJ, Elliott PC, et al. 2019. Pediatric Mild Traumatic Brain Injury and Population Health: An Introduction for Nursing Care Providers [J]. Heart Lung Circ. 28(12):1812-1818.

Zhang Z, Bai J, Huang Y, et al. 2020. Implementation of a clinical nursing pathway for percutaneous coronary intervention: A randomized controlled trial protocol [J]. Medicine (Baltimore). 99(43):e22866.

Zullo AR, Riester MR, Erqou S, et al. 2020. Comparative Effectiveness of Angiotensin II Receptor Blockers and Angiotensin-Converting Enzyme Inhibitors in Older Nursing Home Residents After Myocardial Infarction: A Retrospective Cohort Study [J]. Drugs Aging. 37(10):755-766. 